\title{
Cuantificación de los componentes que afectan el coeficiente de atenuación vertical para irradiancia descendente en el embalse Riogrande II (Colombia)
}

\author{
John Jairo Ramírez R.* , Mónica Cristina Arcila L., Sandra Catalina Sepúlveda A. \\ Grupo de Limnología Básica y Experimental y Biología y Taxonomía Marina, \\ Facultad de Ciencias Exactas y Naturales, Universidad de Antioquia, Medellín, Colombia
}

\begin{abstract}
Resumen
Con el fin de determinar cuál es el componente que más contribuye a la atenuación vertical de la irradiancia descendente $\left[\mathrm{K}_{\mathrm{d}(\mathrm{PAR})}\right]$ en el embalse Ríogrande II, se usó un modelo de regresión lineal múltiple para obtenerla a partir de la suma de los $\mathrm{K}_{\mathrm{d}}$ parciales representados por el agua $\left(\mathrm{K}_{\mathrm{w}}\right)$, la clorofila a $\left(\mathrm{K}_{\mathrm{chl}}\right)$ ), el material inorgánico particulado (tripton, $\left.\mathrm{K}_{\text {tripton }}\right)$, y el fitoplancton en forma de partícula $\left(\mathrm{K}_{\mathrm{p} \text {-fito }}\right)$. Se tomaron muestras de agua con una botella Schindler. La radiación fotosintéticamente activa (PAR) se midió con un cuantómetro Li-cor en cinco estaciones cada diez días a partir de julio de 2002 y hasta julio de 2003. Se cuantificaron los sólidos suspendidos totales, los sólidos suspendidos inorgánicos, los sólidos suspendidos volátiles y la clorofila a. Debido a que el valor de $\mathrm{K}_{\text {tripton }}\left(1,14 \mathrm{~m}^{-1}\right)$ fue el más influyente, se aceptó parcialmente la previsión referente a que en la estación Río Chico arriba la influencia de $\mathrm{K}_{\mathrm{cl} \text {. a }}, \mathrm{K}_{\mathrm{p} \text {-fito }} \mathrm{y} \mathrm{K}_{\text {tripton }}$ sería alta y similar. La ecuación estimada para calcular el coeficiente de atenuación vertical en el embalse fue $\mathrm{K}_{\mathrm{d}(\mathrm{PAR})}=1,32 \mathrm{D}_{\mathrm{sd}}{ }^{-0,93}$, en la que $\mathrm{D}_{\text {sd }}$ explicó el $62,4 \%$ de la variación de $\mathrm{K}_{\mathrm{d}}$. Se consideró que ópticamente el embalse era de tipo T y caso 2, lo que corresponde a un sistema turbio, en el que a pesar de su condición eutrófica y su alta productividad, no fue el fitoplancton sino el tripton la fracción que más capturó los quanta fotosintéticos.
\end{abstract}

Palabras clave: Ríogrande II, radiación fotosintéticamente activa, coeficiente de atenuación vertical para irradiancia descendente, profundidad Secchi, clasificación óptica.

Quantification of components affecting the vertical attenuation coefficient for downward irradiance in Ríogrande II reservoir (Colombia)

\begin{abstract}
In order to determine which component contributes most to the vertical attenuation coefficient for downward irradiance $\left[\mathrm{K}_{\mathrm{d}(\mathrm{PAR})}\right]$ in Ríogrande II reservoir, a multiple linear regression model was used to obtain $\mathrm{K}_{\mathrm{d}(\mathrm{PAR})}$ considering the sum of partial $\mathrm{K}_{\mathrm{d}}$ contributed by water $\left(\mathrm{K}_{\mathrm{w}}\right)$, chlorophyll a $\left(\mathrm{K}_{\mathrm{chl}}\right)$ ), particulated inanimate material (tripton, $\left.\mathrm{K}_{\text {tripton }}\right)$, and phytoplankton-like particles $\left(\mathrm{K}_{\mathrm{p} \text {-phyto }}\right)$. Samples of water were taken with a Schindler bottle. The photosyntetic active radiation (PAR) was measured with a Li-cor quantameter at five sampling stations every 10 days from July 2002 to July 2003. Total suspended solids, inorganic suspended solids, volatile suspended solids, and chlorophyll a were quantified. Given that the $\mathrm{K}_{\text {tripton }}\left(1,14 \mathrm{~m}^{-1}\right)$ value was the highest, the prediction that the influence of $\mathrm{K}_{\text {chl. a, }} \mathrm{K}_{\text {p-phyto. }}$ and $\mathrm{K}_{\text {tripton }}$ would be high and similar at the upstream Chico River station was partially accepted. The equation to estimate $\mathrm{K}_{\mathrm{d}(\mathrm{PAR})}$ for the reservoir was $\mathrm{K}_{\mathrm{d}(\mathrm{PAR})}=1,32 \mathrm{D}_{\text {sd }}^{-0,93}$, with $\mathrm{D}_{\mathrm{sd}}$ explaining $62,4 \%$ of the variation in $\mathrm{K}_{\mathrm{d}}$. Optically, the reservoir was classified as T-type and case 2 , which correspond to a turbid system where despite its eutrophic condition and high productivity, tripton and not phytoplankton was the fraction that harvested more photosynthetic quanta.
\end{abstract}

Key words: Ríogrande II, photosynthetic active radiation, vertical attenuation coefficient for downwelling irradiance, Secchi depth, optical classification.

\section{Introducción}

La luz, entendida como la porción del espectro electromagnético a la cual el ojo humano es sensible, es decir, la región entre los 390 y los $740 \mathrm{msnm}$, pierde intensidad con la profundidad, fenómeno conocido como atenuación y regulado por la composición y concentración de varios componentes que incluyen el agua, las sustancias húmicas, el fitoplancton, la clorofila a, y el tripton (organismos muertos, detritos y sustancias coloidales de origen orgánico o inorgánico) (Roldán \& Ramírez, 2008).

\footnotetext{
*Correspondencia:

John Jairo Ramírez R., johnra77@gmail.com

Recibido: 27 de noviembre de 2014

Aceptado: 17 de marzo de 2015
} 
Al penetrar en el agua, la luz es la causante de los cambios de temperatura que ocasionan la estratificación de la densidad y del establecimiento del límite vertical de la producción primaria conocido como zona eufótica (Deu) o fótica. Para medir la Deu se han diseñado varios instrumentos como los espectroradiómetros, los hidrofotómetros y los cuantómetros. Sin embargo, a pesar de su sencillez y de no ser un instrumento electrónico, el disco de Secchi, de 20 a 30 $\mathrm{cm}$ de diámetro, es el instrumento óptico más antiguo usado para evaluar de manera rápida y simple la transparencia, la profundidad de la zona fótica y los coeficientes de atenuación y de extinción verticales de irradiancia descendente.

Kirk (1994, 2011) demostró que la profundidad Secchi (Dsd) es particularmente sensible a la turbidez, entendida en el sentido de Clack \& Williams (1981), es decir, relacionada con los coloides y los sólidos disueltos; quizás por eso Preisendorfer (1986) estableció que la función primaria del disco de Secchi consistía únicamente en proveer un índice visual simple de la claridad del agua en términos de Dsd, que corresponde a la profundidad con una intensidad de luz entre 5 y $15 \%$ de la iluminación en superficie (Hutchinson, 1957; Vollenweider, 1969).

Uno de los primeros acercamientos en busca de una relación empírica entre la Dsd y el coeficiente de atenuación vertical de la irradiancia descendente $\left(\mathrm{K}_{\mathrm{d}}\right.$ según la terminología de Kirk, 1994, 2011), fue el de Poole \& Atkins (1929), quienes propusieron que en la banda de radiación fotosintéticamente activa (PAR) del espectro lumínico visible se daba una relación aproximadamente inversa entre Dsd y $\mathrm{K}_{\mathrm{d}(\mathrm{PAR})}=$ 1,70/Dsd. Durante mucho tiempo, antes de que se contara con instrumentos electrónicos adecuados, e incluso hoy cuando se cuenta con ellos, esta ecuación se utilizó como una manera rápida de medir la $\mathrm{K}_{\mathrm{d}}$ (Wetzel, 2001). Sin embargo, su aplicación es restringida si se tiene en cuenta que: 1) el valor de Dsd obtenido es equivalente más o menos al $16 \%$ de la luz incidente en superficie (Cole, 1983), y 2) el valor de la constante es de 1,44 según Kirk $(1994,2011)$, aunque Cole (1983), Margalef (1983), Esteves (2011) y Tundisi \& Tundisi (2008) usan el valor de 1,7.

Dado el carácter eutrófico de sus aguas, en embalses como el de Ríogrande II la penetración de la luz está sometida a procesos de atenuación vertical intensos debido a la acción combinada de varios de los factores mencionados antes. Por ello, esta investigación se propuso responder a la siguiente pregunta: ¿En cuál de las estaciones de muestreo del embalse son más importantes los componentes del coeficiente de atenuación vertical de la irradiancia descendente total $\left(\mathrm{K}_{\mathrm{w}}\right.$, $\mathrm{K}_{\text {Cl. a }}, \mathrm{K}_{\text {tripton }} \mathrm{y} \mathrm{K}_{\text {p-fito }}$ ) ? Si la estación Río Chico arriba, como lo muestran las investigaciones de Mazo (2008), FrancoVelásquez (2011), Loaiza, et al. (2011), Vergara (2011), Johnson \& Vahos (2011), Zabala (2013) y PalacioBetancourt (2014), es la más eutrófica del embalse, con los menores valores de profundidad Secchi $($ media $=1,12 \mathrm{~m}$ ) $y$ de $\mathrm{K}_{\mathrm{d}(\mathrm{PAR})}\left(\right.$ media $\left.=1,7 \mathrm{~m}^{-1}\right)$, y la mayor concentración de sólidos suspendidos totales (media $=75,8 \mathrm{mg} / 1$, de los que $87,07 \%$ son disueltos) y de biomasa fitoplanctónica (media $=107,1 \mathrm{mg} \mathrm{Cl}$ a $/ \mathrm{m}^{3}$ ), entonces la previsión es que en ella se incrementa la influencia del $\mathrm{K}_{\mathrm{Cl} \text {. a, }}$, del $\mathrm{K}_{\mathrm{p} \text {-fito }} \mathrm{y}$ del $\mathrm{K}_{\text {tripton }}$. Como un aporte práctico del trabajo, se calculó un $\mathrm{Kd}_{\text {(PAR) }}$ para cada estación de muestreo y para el embalse, al igual que una constante y una ecuación del tipo de la de Poole \& Atkins (1929), lo que permite estimar el $\mathrm{K}_{\mathrm{d}(\mathrm{PAR})}$ para el sistema acuático a partir de la Dsd. Además, se clasificó el embalse desde el punto de vista óptico.

\section{Materiales y métodos}

\section{Área de estudio}

La cuenca del río Grande está localizada en la parte central del departamento de Antioquia, entre los 6 $6^{\circ} 35^{\prime} 57^{\prime \prime}$ y $6^{\circ} 28^{\prime} 79^{\prime \prime} \mathrm{N}$ y los $75^{\circ} 32^{\prime} 17^{\prime \prime}$ y $75^{\circ} 25^{\prime} 85^{\prime \prime} \mathrm{O}$, con un área de captación de $1.294 \mathrm{~km}^{2}(=129.400 \mathrm{ha})$, temperaturas entre los 14 y $18{ }^{\circ} \mathrm{C}$, dos períodos lluviosos, uno entre abril y junio y otro entre septiembre y noviembre, y mayores valores de humedad entre octubre y noviembre, con promedios cercanos al $83 \%$. Las épocas secas van de diciembre a marzo y de julio a agosto, siendo la primera más seca que la segunda (EEPPM, 1989).

Por su parte, el embalse Ríogrande II se localiza en las cuencas de drenaje de los ríos Grande y Chico en las que encuentran asentados los municipios de Belmira, Don Matías, Entrerríos, San Pedro y Santa Rosa de Osos. Se sitúa a una altura media de $2.150 \mathrm{msnm}$, con un volumen de 253 millones de $\mathrm{m}^{3}$, una profundidad máxima de $59 \mathrm{~m}$, un tiempo medio de residencia de 72,8 días, y un caudal de $35 \mathrm{~m}^{3} / \mathrm{s}$ en la captación. La presa, de 2,8 millones de $\mathrm{m}^{3} \mathrm{y}$ 65 metros de altura, está provista de un vertedero a 2.270 msnm que crea el embalse que inunda un área aproximada de 10.100 ha (EEPPM, 1989).

La principal práctica económica en la región donde se localiza el embalse es la agropecuaria, destacándose los cultivos y la explotación lechera y porcina, actividades estas que demandan alto consumo de plaguicidas y abonos orgánicos y potencian la eutrofización del cuerpo de agua (Londoño \& Villegas, 1996). Además, en el río Chico se practica la minería de aluvión, la cual constituye una fuente importante de sedimentos para esta estación y para el embalse en general (EEPPM, 1984).

\section{Diseño muestral}

Se ubicaron en el embalse cinco estaciones de muestreo (Figura 1):

- Estación Presa (1): está situada a un kilómetro de la presa, presenta las mayores profundidades del embalse $(35 \mathrm{y}$ $40 \mathrm{~m}$ en promedio), y constituye un punto que permite caracterizar la zona limnética del embalse.

- Estación río Grande (2): se localiza aguas abajo del río Grande, en el límite de la entrada hacia su represamiento, 

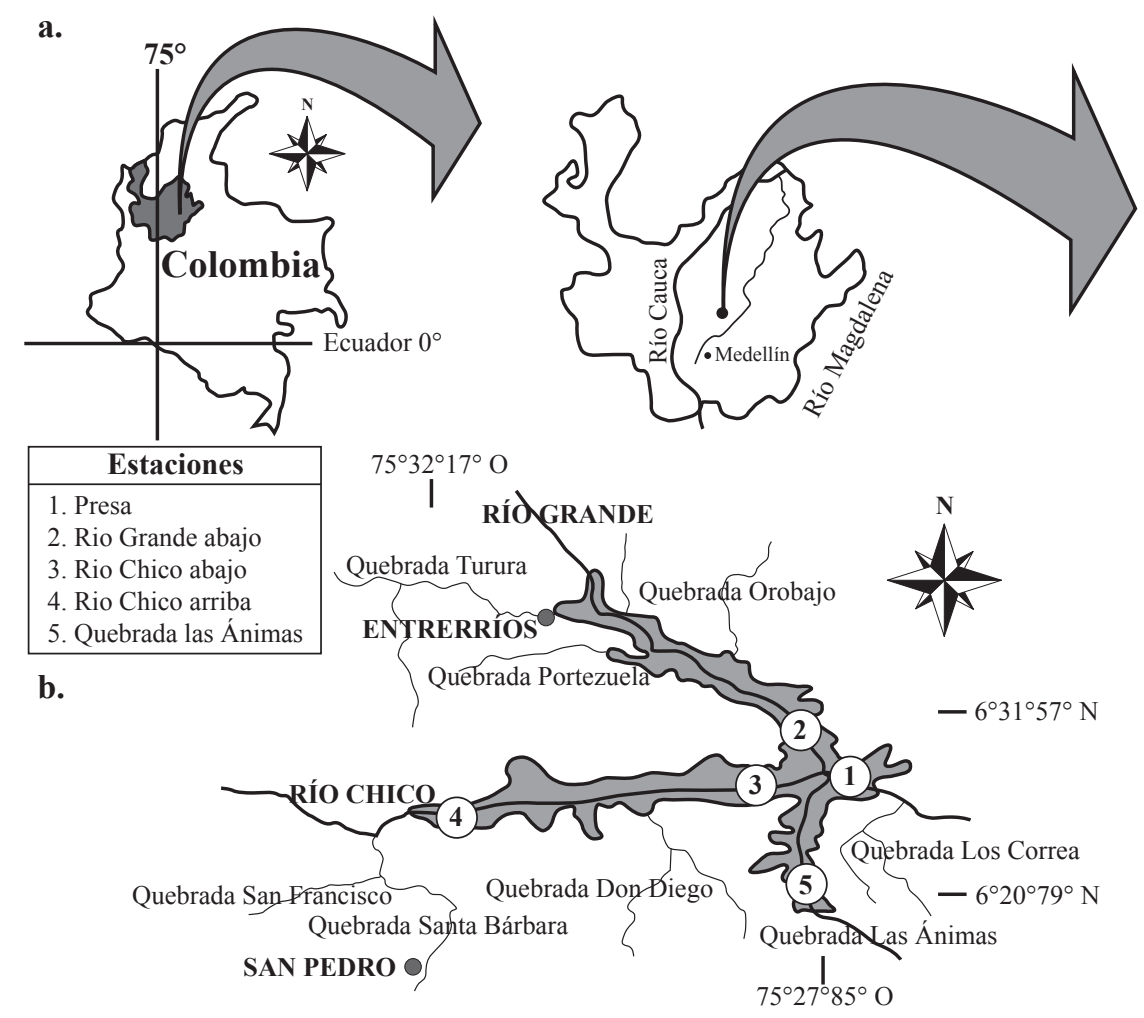

Figura 1. (A) Ubicación del embalse Ríogrande II en el contexto regional. (B) Localización de las estaciones de muestreo en el embalse.

tiene una profundidad que varía entre 30 y $35 \mathrm{~m}$ y representa una zona transicional del embalse. El río Grande se encuentra cerca al municipio de Entrerríos y en él desembocan las quebradas Orobajo, Portezuela y Turura.

- Estaciones río Chico abajo y río Chico arriba (3 y 4): están localizadas en la parte alta del río, cerca al sitio de entrada de los vertimientos provenientes del río Chico al embalse. La estación Chico abajo representa también una zona transicional del embalse, con una profundidad entre 25 y $30 \mathrm{~m}$. La profundidad de la estación Chico arriba fluctúa entre 7 y $18 \mathrm{~m}$. El río Chico recibe aportes de los municipios de Belmira y San Pedro.

- Estación quebrada Las Ánimas (5): está ubicada a un kilómetro de la torre de captación en la entrada de la quebrada Las Ánimas; representa la zona que aporta el menor caudal al embalse y posee una de las menores profundidades (entre 12 y $15 \mathrm{~m}$ ).

La torre de captación se localiza en un brazo del embalse que sube por la cuenca de la quebrada Las Ánimas. El agua se capta en la torre mediante un sistema de cuatro compuertas rectangulares distribuidas en dos niveles, así: entre los 2.257,53 y los 2.254,03 msnm (compuerta superior), se capta un caudal máximo de $6,4 \mathrm{~m}^{3} / \mathrm{s}$ para la planta de tratamiento de aguas Manantiales; entre los $2.249,47$ y los 2.245,97 msnm (compuertas inferiores), se capta un caudal máximo de $40 \mathrm{~m}^{3} / \mathrm{s}$ para la central hidroeléctrica La Tasajera a través de dos compuertas, y por la compuerta restante se capta en ocasiones para la planta Manantiales (Franco-Velásquez, et al., 2010).

Franco-Velásquez (2011) determinó que el caudal de río Grande es, en promedio, tres veces mayor que el del río Chico; por ello, al entrar al cuerpo de agua el río Grande reparte su volumen entre el brazo del río Chico y el de la quebrada Las Ánimas. Las aguas del río Grande se internan en el río Chico hasta localizarse a $4 \mathrm{~km}$ de su cola, aumentando así su tiempo de residencia. Sobre el brazo de la quebrada Las Ánimas, el río Grande viaja directamente hasta la torre de captación a nivel de la compuerta superior. Por su parte, el río Chico ingresa al embalse como una corriente de fondo difícil de rastrear, pues se mezcla rápidamente con el agua del embalse. En las proximidades de la torre de captación, dicha pluma se orienta hacia la compuerta inferior debido al caudal extraído para la generación de energía en la central de La Tasajera.

El embalse permanece estratificado a lo largo del año. Las variaciones de temperatura a lo largo del año en la capa superficial oscilan entre $19,5-23,7^{\circ} \mathrm{C}$ (media: $21,6 \pm 1,35^{\circ} \mathrm{C}$ ). Esta capa superficial presenta variaciones en el espesor que están entre los 3,0 y los 6,0 m de profundidad. La zona del hipolimnio se caracteriza por presentar pocas variaciones a lo largo del año y su temperatura oscila entre los 17,4 y $\operatorname{los} 18,2{ }^{\circ} \mathrm{C}$ (media: 17,4 $\pm 0,92$ ). Esta estructura térmica 
está fuertemente asociada a la dinámica de la corriente de densidad del río Grande, que entra al cuerpo de agua como una corriente intrusiva a una profundidad entre 5 y $10 \mathrm{~m}$, modificando el decaimiento exponencial de la temperatura en la columna de agua (Franco-Velásquez, 2011).

\section{Métodos}

La presente investigación se basa en los datos recolectados en el marco del proyecto "Caracterización limnológica del embalse Ríogrande II, Antioquia, Colombia" mediante muestreos adelantados cada diez días en cada una de las cinco estaciones mencionadas entre julio de 2002 y julio de $2003(\mathrm{n}=180 ; \mathrm{n}$ en cada estación de muestreo $=36)$. Las muestras de agua se extrajeron con una botella Schindler de 5 litros, integrando las recogidas en la superficie, la mitad de la zona fótica y su fondo.

La Dsd se midió en cada estación y en cada muestreo con un disco de Secchi (diámetro $=0,20 \mathrm{~m}$, bandas blancas y negras alternadas).

Con un datalogger Li-cor equipado con un sensor esférico 193SB se efectuaron mediciones de PAR cada $0,50 \mathrm{~m}$ en cada estación de muestreo; por ello, el $\mathrm{K}_{d}$ hallado en esta investigación debe entenderse como el $\mathrm{K}_{\mathrm{d}}(\mathrm{PAR})$ de la irradiación descendente. Este se calculó a partir de la pendiente del perfil obtenido una vez que los datos de luz (Io) fueron transformados logarítmicamente.

La regresión $\mathrm{K}_{\mathrm{d}}(\mathrm{PAR})$ medido- $\mathrm{K}_{\mathrm{d}}(\mathrm{PAR})$ calculado de la figura 4 se efectuó con base en los valores medios de cada estación $(\mathrm{n}=36)$, por eso en dicha figura aparecen solo cinco valores (uno por estación de muestreo).

Los sólidos suspendidos totales $\left(\mathrm{SS}_{\mathrm{T}}\right)$ se obtuvieron al establecer la diferencia entre el peso de un filtro de fibra de vidrio $(\varphi=0,45 \mu \mathrm{m})$ antes y después de la filtración de un volumen conocido de agua. Antes de ser pesado, el filtro se secó e incineró a $550{ }^{\circ} \mathrm{C}$ en una mufla durante 24 horas para determinar los sólidos suspendidos inorgánicos o fijos $\left(\mathrm{SS}_{\mathrm{mp} \text {-inorg }}\right)$ y los sólidos suspendidos volátiles $\left(\mathrm{SS}_{\text {mop }}\right)$. Las fórmulas para la determinación de la concentración de los tipos de sólidos mencionados $\left(\mathrm{en} \mathrm{mg} / \mathrm{m}^{3}\right.$ ) se determinaron según los métodos estandarizados (Standard Methods) (APHA, AWWA y WPCF, 1995).

La clorofila a activa ( $\mathrm{Cl}$. a) se extrajo con etanol caliente al $95 \%$; las muestras se acidificaron usando $\mathrm{HCl} 0,1 \mathrm{~N}$ mediante el método espectrofotométrico. Las ecuaciones empleadas para obtener la concentración de clorofila a en $\mathrm{mg} / \mathrm{m}^{3}$ fueron las sugeridas por Sartory \& Grobbellaar (1984).

Los $\mathrm{SS}_{\mathrm{p}-\text { fito }}$ se estimaron como $100(\mathrm{Cl}$. a), según lo sugerido en Reynolds (1984). Esta ecuación se obtiene a partir de la regresión SS - Cl. a, cuya pendiente multiplicada por 100 representa la contribución porcentual de la biomasa fitoplanctónica a los sólidos suspendidos.
La contribución de los sólidos suspendidos del tripton $\left(\mathrm{SS}_{\text {tripton }}\right)$ se valoró a partir de la ecuación $\mathrm{SS}_{\mathrm{T}}-\mathrm{SS}_{\mathrm{p}-\text { fito }}=$ $\left[\mathrm{SS}_{\mathrm{T}}-100(\mathrm{Cl} . \mathrm{a})\right]$.

Los componentes de $\mathrm{K}_{d}(\mathrm{PAR})$ se valoraron de acuerdo al método de regresión lineal simple propuesto en Reynolds (1984).

Debido a que los ácidos húmicos o compuestos coloreados no se midieron en ninguna de las estaciones del embalse, la contribución de $\mathrm{K}_{\text {Gilvin }}\left(\mathrm{K}_{\mathrm{G}}\right)$ al $\mathrm{K}_{\mathrm{d}}(\mathrm{PAR})$ no se consideró. Se sabe que las sustancias amarillas disueltas en los lagos provienen del material húmico soluble lixiviado desde los suelos en el área de la cuenca, o de la descomposición del material vegetal dentro del cuerpo de agua (algas y plantas acuáticas principalmente). Dado que en el lago no se registra la presencia de plantas acuáticas, y que la contribución del fitoplancton es baja, se asumió que la mayor contribución de materia orgánica disuelta coloreada (gilvin) se originaba en la cuenca y no se la valoró; sin embargo, por las condiciones de dicha cuenca es de esperar que sea alta, lo cual recalca la necesidad de estimar en próximas publicaciones la contribución del $\mathrm{K}_{\mathrm{G}}$ al $\mathrm{K}_{\mathrm{d}}(\mathrm{PAR})$ de este embalse.

\section{Análisis estadístico}

En todas las regresiones simples realizadas, la variable de respuesta o dependiente fue $\mathrm{K}_{\mathrm{d}(\mathrm{PAR})}$, y las independientes o causales fueron $\mathrm{SS}_{\mathrm{T}}, \mathrm{SS}_{\text {tripton }}, \mathrm{K}_{\text {dcal }}$ y Dsd. Las relaciones $\mathrm{SS}_{\mathrm{T}}-\mathrm{K}_{\mathrm{d}(\mathrm{PAR})}$ (Figura 2), $\mathrm{SS}_{\text {tripton }}-\mathrm{K}_{\mathrm{d}(\mathrm{PAR})}$ (Figura 3, Tabla 1), $\mathrm{K}_{\mathrm{d}(\mathrm{PAR}) \mathrm{cal}}-\mathrm{K}_{\mathrm{d}(\mathrm{PAR}) \text { med }}$ (Figura 4) y Dsd $-\mathrm{K}_{\mathrm{d}(\mathrm{PAR})}$ (Figura 5), se obtuvieron utilizando modelos de correlación y regresión de los tipos lineal simple y exponencial simple. El tipo de ecuación más ajustado a los datos de las relaciones se determinó de acuerdo al valor del coeficiente de determinación de Pearson $\left(\mathrm{R}^{2}\right)$.

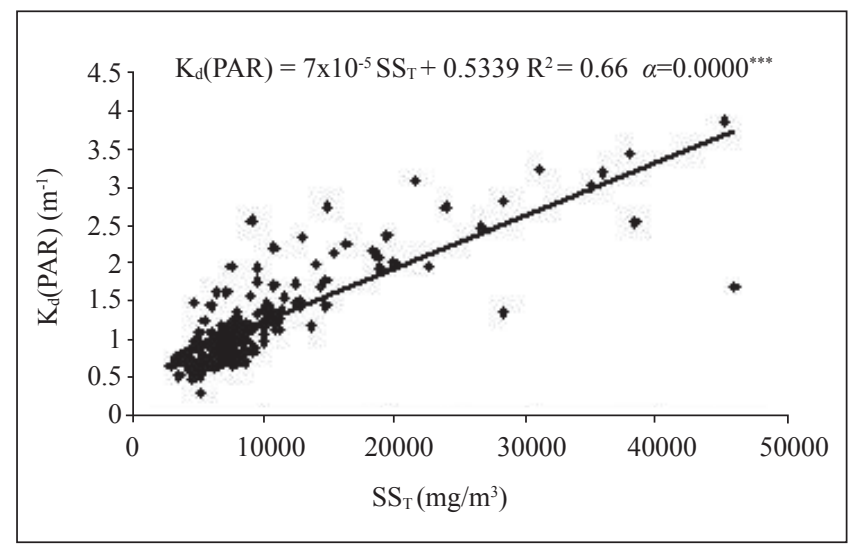

Figura 2. Regresión lineal simple entre la concentración de sólidos suspendidos totales $\left(\mathrm{SS}_{\mathrm{T}}\right)$ y el coeficiente de atenuación vertical total para irradiancia descendente $\left[\mathrm{K}_{\mathrm{d}}(\mathrm{PAR})\right]$ en el embalse Ríogrande II 


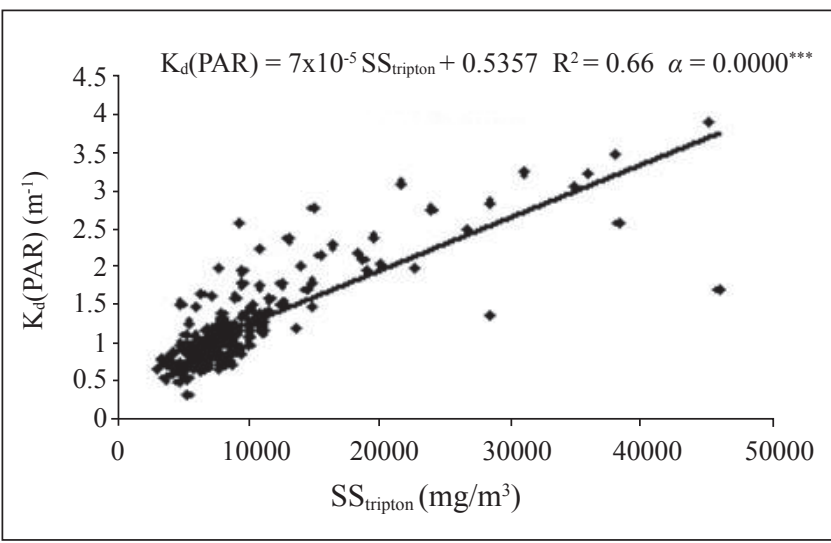

Figura 3. Regresión lineal simple entre la concentración de sólidos suspendidos del tripton ( $\mathrm{SS}_{\text {tripton }}$ ) y $\mathrm{K}_{\mathrm{d}(\mathrm{PAR})}$ del embalse Ríogrande II

Tabla 1. Ecuaciones obtenidas para las regresiones lineales simples $\mathrm{K}_{\mathrm{d}(\mathrm{PAR})}-\mathrm{SS}_{\text {tripton }} \mathrm{y} \mathrm{K}_{\mathrm{d}(\mathrm{PAR})}-\mathrm{SS}_{\mathrm{T}}$, con sus respectivos coeficientes de determinación de Pearson $\left(\mathrm{R}^{2}\right)$, errores estándar (EE) y valores de significancia $(\alpha)$ en cada una de las estaciones de muestreo

\begin{tabular}{llccc}
\hline Estación & \multicolumn{1}{c}{ Ecuación } & $\mathbf{R}^{2}$ & $\mathbf{E E}$ & $\mathrm{a}$ \\
\hline \multirow{2}{*}{ Presa } & $\mathrm{K}_{\mathrm{d}}(\mathrm{PAR})=8 \times 10^{-5} \mathrm{SS}_{\text {tripton }}+0,42$ & 0,41 & 0,2300 & 0,0000 \\
& $\mathrm{~K}_{\mathrm{d}}(\mathrm{PAR})=7 \times 10^{-5} \mathrm{SS}_{\mathrm{T}}+0,63$ & 0,41 & 0,2300 & 0,0293 \\
\hline \multirow{2}{*}{$\begin{array}{l}\text { Chico } \\
\text { arriba }\end{array}$} & $\mathrm{K}_{\mathrm{d}}(\mathrm{PAR})=7 \times 10^{-5} \mathrm{SS}_{\text {tripton }}+0,63$ & 0,82 & 0,3999 & 0,0000 \\
Chico & $\mathrm{K}_{\mathrm{d}}(\mathrm{PAR})=9 \times 10^{-5} \mathrm{SS}_{\mathrm{T}}+0,34$ & 0,82 & 0,3997 & 0,0000 \\
abajo & $\mathrm{K}_{\mathrm{d}}(\mathrm{PAR})=9 \times 10^{-5} \mathrm{SS}_{\text {tripton }}+0,34$ & 0,42 & 0,2300 & 0,0000 \\
\multirow{2}{*}{ Ánimas } & $\mathrm{K}_{\mathrm{d}}(\mathrm{PAR})=4 \times 10^{-5} \mathrm{SS}_{\mathrm{T}}+0,73$ & 0,42 & 0,2300 & 0,0000 \\
& $\mathrm{~K}_{\mathrm{d}}(\mathrm{PAR})=4 \times 10^{-5} \mathrm{SS}_{\text {tripton }}+0,73$ & 0,33 & 0,450 & 0,0004 \\
\hline \multirow{2}{*}{$\begin{array}{l}\text { Río } \\
\text { Grande }\end{array}$} & $\mathrm{K}_{\mathrm{d}}(\mathrm{PAR})=1 \times 10^{-4} \mathrm{SS}_{\mathrm{T}}+0,21$ & 0,33 & 0,4500 & 0,0004 \\
\hline & $\mathrm{K}_{\mathrm{d}}(\mathrm{PAR})=1 \times 10^{-4} \mathrm{SS}_{\text {tripton }}+0,21$ & 0,75 & 0,2800 & 0,0000 \\
\hline & $\mathrm{K}_{\mathrm{d}}(\mathrm{PAR})=1 \times 10^{-4} \mathrm{SS}_{\text {tripton }}+0,21$ & 0,75 & 0,2800 & 0,0000 \\
\hline
\end{tabular}

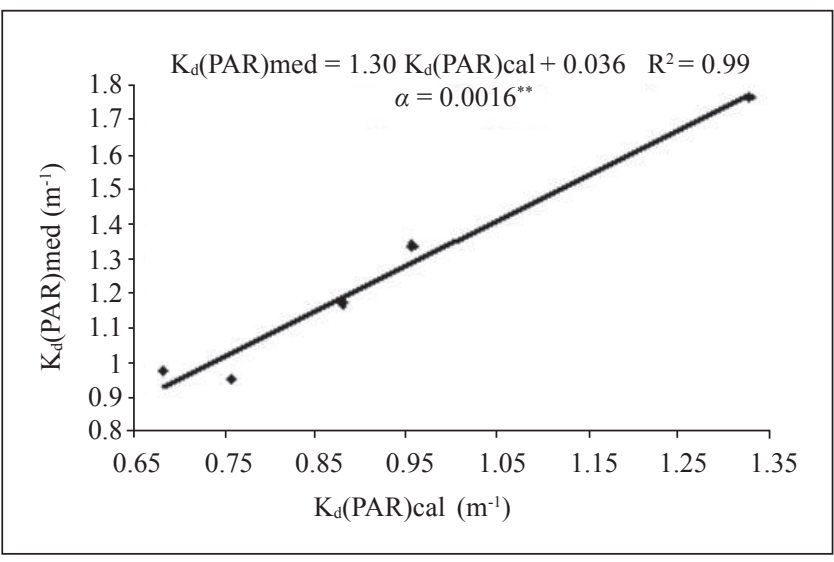

Figura 4. Regresión lineal simple entre los valores de $\mathrm{K}_{\mathrm{d}(\mathrm{PAR})}$ calculados y los $\mathrm{K}_{\mathrm{d}(\mathrm{PAR})}$ medios para las estaciones de muestreo ( $\mathrm{n}=$ 36 datos por estación de muestreo)

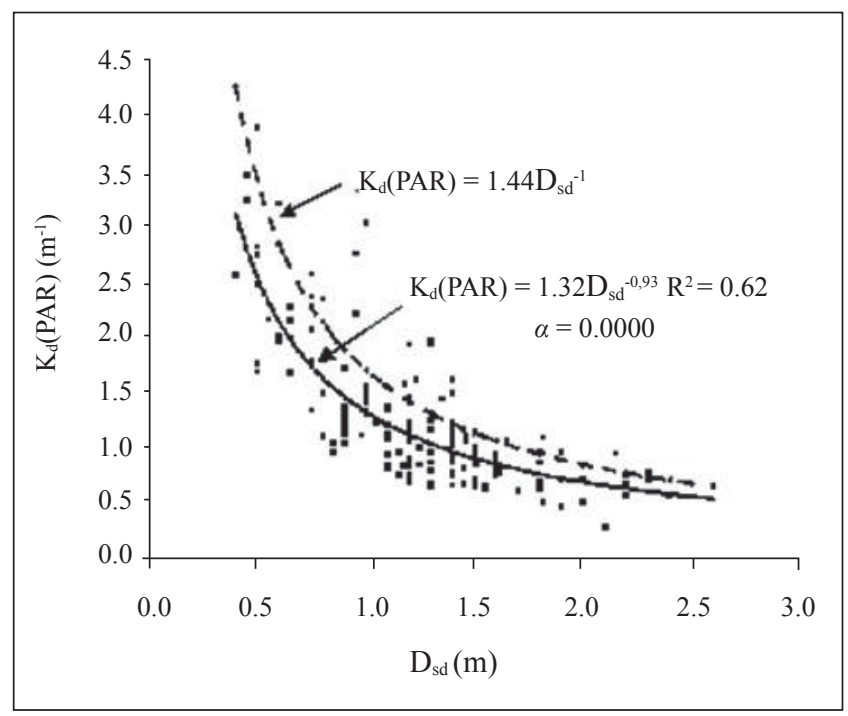

Figura 5. Regresión exponencial simple entre la distancia Secchi (Dsd) y el coeficiente de atenuación vertical de luz para irradiancia descendente $\left[\left(\mathrm{K}_{d}(\mathrm{PAR})\right]\right.$. La línea continua indica la ecuación resultante y la línea discontinua indica los resultados de la ecuación propuesta por Poole \& Atkins (1929).

\section{Resultados}

Los valores de Dsd variaron entre 0,4 y 2,6 m; los mayores se presentaron en Presa, Chico abajo y Las Ánimas; en Chico arriba y río Grande se obtuvieron los menores valores.

El coeficiente $\mathrm{K}_{\mathrm{d}(\mathrm{PAR})}$ osciló para todo el embalse entre 0,29 y $3,88 \mathrm{~m}^{-1}$. Los valores más bajos se obtuvieron en las estaciones Presa, Chico abajo y Las Ánimas, y los más altos en Chico arriba y río Grande.

Los $\mathrm{SS}_{\mathrm{T}}$ fluctuaron en un rango entre 2.999 y $46.000 \mathrm{mg} / \mathrm{m}^{3}$ y la clorofila $a$, entre 0,93 y $600,05 \mathrm{mg} / \mathrm{m}^{3}$.

El patrón general de comportamiento evidenciado en la tabla 2 muestra que las estaciones Chico arriba y Las Ánimas registraron los mayores valores medios y de coeficiente de variación $(\mathrm{CV})$ para el $\mathrm{K}_{\mathrm{d}(\mathrm{PAR})}$, la clorofila $a$, la concentración de sólidos suspendidos totales, los del tripton y los del fitoplancton como partícula. En Presa, Chico abajo y río Grande los valores para todas las variables fueron menores, excepto para Dsd, que a simple vista pareció tener un comportamiento similar en varias estaciones. Estos sitios también registraron los menores coeficientes de variación en la mayoría de los casos.

\section{Descomposición de $K_{d(P A R)}$ para la estimación del $K_{d(P A R)}$ calculado [ $K_{\text {dcal(PAR) }}$ ] del embalse Ríogrande II $(n=180)$}

Para descomponer el $\mathrm{K}_{\mathrm{d}(\mathrm{PAR})}$ se utilizó el modelo de regresión lineal múltiple propuesto por Reynolds (1984). Dicho modelo corresponde a la sumatoria de $\operatorname{los} \mathrm{K}_{\mathrm{d}}$ parciales aportados por la clorofila $\underline{a}\left(\mathrm{~K}_{\mathrm{Cl} \text { a a }}\right)$, el material inorgánico particulado $\left(\mathrm{K}_{\text {tripton }}\right)$, el fitoplancton como partícula $\left(\mathrm{K}_{\mathrm{p}-\mathrm{fito}}\right) \mathrm{y}$ 
Tabla 2. Valores de la media aritmética y del coeficiente de variación $(\mathrm{CV} \%)$ para el coeficiente de atenuación total [ $\left.\mathrm{K}_{\mathrm{d}(\mathrm{PAR})}\right]$, la profundidad Secchi (Dsd), la clorofila a $(\mathrm{Cl}$ a $)$, los sólidos suspendidos totales $\left(\mathrm{SS}_{\mathrm{T}}\right)$, los sólidos suspendidos del tripton $\left(\mathrm{SS}_{\text {tripton }}\right)$ y los sólidos suspendidos del fitoplancton $\left(\mathrm{SS}_{\mathrm{p} \text {-fito }}\right.$ ) para cada una de las estaciones

\begin{tabular}{|c|c|c|c|c|c|c|}
\hline \multirow{2}{*}{ Variable } & \multirow{2}{*}{ Estadístico } & \multicolumn{5}{|c|}{ Estación } \\
\hline & & Presa & Chico Arriba & Chico Abajo & Ánimas & Río Grande \\
\hline \multirow{2}{*}{$\mathrm{K}_{\mathrm{d}(\mathrm{PAR})}\left(\mathrm{m}^{-1}\right)$} & Media & 1,0 & 1,7 & 0,9 & 1,2 & 1,2 \\
\hline & CV (\%) & 29,8 & 52,6 & 31,1 & 46,1 & 42,0 \\
\hline \multirow{2}{*}{$\mathrm{D}_{\mathrm{sd}}(\mathrm{m})$} & Media & 1,4 & 1,1 & 1,4 & 1,4 & 1,2 \\
\hline & CV (\%) & 24,9 & 39,5 & 28,7 & 38,3 & 31,9 \\
\hline \multirow{2}{*}{ Cl. $\underline{\mathrm{a}}\left(\mathrm{mg} / \mathrm{m}^{3}\right)$} & Media & 18,0 & 111,9 & 23,1 & 24,8 & 38,0 \\
\hline & CV (\%) & 56,3 & 141,7 & 88,7 & 117,8 & 112,8 \\
\hline \multirow{2}{*}{$\mathrm{SS}_{\mathrm{T}}\left(\mathrm{mg} / \mathrm{m}^{3}\right)$} & Media & 7395,0 & 16330,5 & 7193,2 & 10486,4 & 9198,1 \\
\hline & CV (\%) & 33,8 & 71,1 & 29,6 & 79,1 & 42,9 \\
\hline \multirow{2}{*}{$\mathrm{SS}_{\text {tripton }}\left(\mathrm{mg} / \mathrm{m}^{3}\right)$} & Media & 7394,2 & 16319,3 & 7190,9 & 10483,9 & 9194,3 \\
\hline & CV (\%) & 33,8 & 71,0 & 29,6 & 79,1 & 42,9 \\
\hline \multirow{2}{*}{$\mathrm{SS}_{\mathrm{p} \text {-fito }}\left(\mathrm{mg} / \mathrm{m}^{3}\right)$} & Media & 1,8 & 11,2 & 2,3 & 2,5 & 3,8 \\
\hline & CV (\%) & 56,1 & 141,7 & 88,7 & 118,1 & 0,1 \\
\hline
\end{tabular}

Las cifras en negrilla representan los valores menores o los mayores de la variable correspondiente entre las cinco estaciones de muestreo.

el agua $\left(\mathrm{K}_{w}\right)$; para este último se utilizó un valor de $0,09 \mathrm{~m}^{-1}$, correspondiente a la media aritmética de los valores $0,08 \mathrm{y}$ 0,11 $\mathrm{m}^{-1}$ citados por Reynolds (1979).

$$
\mathrm{K}_{\mathrm{d}(\mathrm{PAR})}=\mathrm{K}_{\mathrm{w}}+\mathrm{K}_{\mathrm{Cl} \text {. a }}+\mathrm{K}_{\text {tripton }}+\mathrm{K}_{\mathrm{p}-\text { fito }}
$$

El primer paso consistió en hacer la regresión entre $\mathrm{SS}_{\mathrm{T}}-$ $\mathrm{K}_{\mathrm{d}(\mathrm{PAR})}$ (Figura 2). En la ecuación obtenida el valor 0,5339 $\mathrm{m}^{-1}$ es el intercepto y corresponde al valor de $\mathrm{K}_{\mathrm{w}}+\mathrm{K}_{\mathrm{Cl} . \mathrm{a}}$; se despejó el valor del $\mathrm{K}_{\mathrm{Cl} \text { a }}$ de la ecuación de la siguiente forma:

$$
\begin{gathered}
\mathrm{K}_{\mathrm{Cl.} \mathrm{a}}=0,5339 \mathrm{~m}^{-1}-\mathrm{K}_{\mathrm{w}} \\
\mathrm{K}_{\text {Cl. a }}=0,5339 \mathrm{~m}^{-1}-0,09 \mathrm{~m}^{-1} \\
\mathrm{~K}_{\text {Cl. a }}=0,4439 \mathrm{~m}^{-1}
\end{gathered}
$$

El siguiente paso consistió en realizar la regresión entre $\mathrm{SS}_{\text {tripton }}-\mathrm{K}_{\mathrm{d}(\mathrm{PAR})}$ (Figura 3). En ella, el valor del intercepto $(0,5357)$ corresponde a $\mathrm{K}_{\mathrm{w}}+\mathrm{K}_{\mathrm{Cl.} \mathrm{a}}+\mathrm{K}_{\mathrm{p}-\text { fito }}$. Puesto que ya se conoce el valor de $\mathrm{K}_{\mathrm{w}}+\mathrm{K}_{\mathrm{Cl} \text { a a }}$ es posible conocer el valor del $\mathrm{K}_{\mathrm{p} \text {-fito }}$ como el resultado de la operación $\left[0,5357-\left(\mathrm{K}_{\mathrm{w}}\right.\right.$ $+\mathrm{K}_{\mathrm{Cl} . \mathrm{a}}^{\mathrm{a}}$ )]:

$$
\begin{gathered}
\mathrm{K}_{\mathrm{p}-\text { fito }}=0,5357 \mathrm{~m}^{-1}-\left(\mathrm{K}_{\mathrm{w}}+\mathrm{K}_{\mathrm{Cl} . \mathrm{a}}\right) \\
\mathrm{K}_{\mathrm{p} \text {-fito }}=0,5357 \mathrm{~m}^{-1}-(0,09+0,4439) \\
\mathrm{K}_{\mathrm{p}-\text { fito }}=0,5357 \mathrm{~m}^{-1}-0,5339 \mathrm{~m}^{-1} \\
\mathrm{~K}_{\mathrm{p}-\text { fito }}=0,00181=\sim 0,002 \mathrm{~m}^{-1}
\end{gathered}
$$

A partir de esta misma ecuación se puede obtener también el valor de $\mathrm{K}_{\text {tripton, }}$ que corresponde al valor de la pendiente $(7 \mathrm{x}$
$10^{-5}$ ) hallada en la figura 2, multiplicado por la concentración media de $\mathrm{SS}_{\text {tripton }}$ para el embalse.

$$
\begin{gathered}
\mathrm{K}_{\text {tripton }}=7 \times 10^{-5} \mathrm{~m}^{2} / \mathrm{mg} \times 10116,52 \mathrm{mg} / \mathrm{m}^{3} \\
\mathrm{~K}_{\text {tripton }}=0,708 \mathrm{~m}^{-1}
\end{gathered}
$$

Por último, se hace posible la estimación del $\mathrm{K}_{\mathrm{T}}$ calculado $\left[\left(\mathrm{K}_{\text {dcal(PAR) }}\right]\right.$ para el embalse a partir de la sumatoria de los $\mathrm{K}_{\mathrm{d}}$ parciales,

$$
\begin{gathered}
\mathrm{K}_{\text {dcal(PAR) }}=\mathrm{K}_{\mathrm{w}}+\mathrm{K}_{\mathrm{Cl} \cdot \mathrm{a}}+\mathrm{K}_{\text {tripton }}+\mathrm{K}_{\mathrm{p} \text {-fito }} \\
\mathrm{K}_{\text {dcal(PAR) }}=0,09 \mathrm{~m}^{-1}+0,4439 \mathrm{~m}^{-1}+0,708 \mathrm{~m}^{-1}+0,002 \mathrm{~m}^{-1}= \\
1,2439=\sim 1,24 \mathrm{~m}^{-1}
\end{gathered}
$$

De igual forma, pero con un menor número de datos, se procedió para cada estación de muestreo $(\mathrm{n}=36)$.

En las figuras 2 y 3 se observa que las correlaciones entre $\mathrm{K}_{\mathrm{d}(\mathrm{PAR})}$ y las variables $\mathrm{SS}_{\mathrm{T},}$ y $\mathrm{SS}_{\text {tripton }}$ obtuvieron un $\mathrm{R}^{2}$ de 0,66 , ambas altísimamente significativas $(\alpha £ 0,005)$.

La estación Chico arriba mostró los mejores ajustes para las regresiones $\mathrm{K}_{\mathrm{d}(\mathrm{PAR})}-\mathrm{SS}_{\mathrm{T}}$ y $\mathrm{K}_{\mathrm{d}(\mathrm{PAR})}-\mathrm{SS}_{\text {tripton}}$, seguida por río Grande, que para las mismas regresiones presentó un $\mathrm{R}^{2}$ de $75,0 \%$. Las demás estaciones presentaron valores de $\mathrm{R}^{2}$ relativamente similares (Tabla 1 ).

Los valores de los $\mathrm{K}_{\mathrm{d}}$ parciales para cada una de las estaciones y el valor calculado para el embalse se muestran en la tabla 3. En ella se observa que el $\mathrm{K}_{\mathrm{p} \text {-fito }}$ y el $\mathrm{K}_{\mathrm{w}}$ presentaron la menor contribución para todos los sitios de muestreo. Igualmente, se ve en esta tabla que para el $\mathrm{K}_{\mathrm{Cl} \text {. a }}$, las estaciones con mayores 
Tabla 3. Contribución de los $\mathrm{K}_{\mathrm{d}}$ parciales y valor del $\mathrm{K}_{\mathrm{d}(\mathrm{PAR})}$ calculado en cada estación de muestreo y en el embalse

\begin{tabular}{lccccc}
\hline Estación & $\mathbf{K}_{\text {tripton }}$ & $\mathbf{K}_{\mathrm{Cl} \text { a }}$ & $\mathbf{K}_{\mathrm{w}}$ & $\mathbf{K}_{\mathrm{p} \text {-fito }}$ & $\begin{array}{c}\mathbf{K}_{\mathrm{d}(\text { PAR })} \\
\text { calculado }\end{array}$ \\
\hline Embalse & 0,71 & 0,44 & 0,09 & 0,0002 & 1,24 \\
Presa & 0,59 & 0,33 & 0,09 & 0,0002 & 1,01 \\
Chico arriba & 1,14 & 0,49 & 0,09 & 0,0004 & 1,72 \\
\hline Chico abajo & 0,65 & 0,21 & 0,09 & 0,0004 & 0,95 \\
Ánimas & 0,42 & 0,68 & 0,09 & 0,0004 & 1,19 \\
\hline Río Grande & 0,92 & 0,15 & 0,09 & 0,0005 & 1,16 \\
\hline
\end{tabular}

aportes fueron Las Ánimas y Chico arriba y la de menor aporte fue río Grande. Se observa, además, que para el $\mathrm{K}_{\text {tripton, }}$, la mayor participación se obtuvo en Chico arriba y río Grande y que Las Ánimas brindó el menor valor. La misma tabla 3 muestra que una vez calculado el $\mathrm{K}_{\mathrm{d}(\mathrm{PAR})}$, la estación con el mayor coeficiente de atenuación fue Chico arriba, seguida por Las Ánimas y río Grande; Chico abajo y Presa fueron las que presentaron los menores valores de $\mathrm{K}_{\mathrm{dcal}(\mathrm{PAR})}$. Para el embalse, el valor calculado de $\mathrm{K}_{\mathrm{d}(\mathrm{PAR})}$ fue de $1,24 \mathrm{~m}^{-1}$.

Como se puede ver en la figura 4, la relación entre los $\mathrm{K}_{\mathrm{d}(\mathrm{PAR})}$ medidos y los $\mathrm{K}_{\mathrm{d}(\mathrm{PAR})}$ calculados fue muy significativa y se ajustó muy rigurosamente a un modelo lineal $(\alpha=$ $0,0016)$; el $\mathrm{K}_{\mathrm{d}(\mathrm{PAR})}$ calculado explicó el $99 \%$ de la variación del $\mathrm{K}_{\mathrm{d}(\mathrm{PAR})}$ medido. Las estaciones donde el material no fitoplanctónico se absorbió mayormente fueron Presa $(0,73)$ y Chico abajo $(0,69)$.

En Chico arriba, río Grande y Chico abajo, el $\mathrm{K}_{\mathrm{d}}$ parcial correspondiente a la fracción de $\mathrm{SS}_{\text {tripton }}$ fue el que más contribuyó a la atenuación de la luz, con un porcentaje del 79,3\% para río Grande seguido por los porcentajes de $68,4 \%$ y $66,3 \%$ para Chico abajo y Chico arriba, respectivamente. El $\mathrm{K}_{\mathrm{d}}$ correspondiente a la fracción de la $\mathrm{Cl}$. a fue mayor para las estaciones Las Ánimas (porcentaje de $57,1 \%$ ) y Presa, con $32,7 \%$. En todas las estaciones $\mathrm{K}_{\mathrm{w}}$ contribuyó con un porcentaje bastante bajo, que varió entre $5,2 \%$ (Chico arriba) y 9,5\% (Chico abajo). La fracción de $\mathrm{K}_{\mathrm{d}}$ que contribuyó en menor proporción al $\mathrm{K}_{\mathrm{d}(\mathrm{PAR})}$ fue la del fitoplancton, con un porcentaje medio del $0,03 \%$.

\section{Discusión}

La atenuación de la luz aumenta por la resuspensión de sedimentos durante la circulación del agua, o por la entrada de ríos o quebradas que aportan gran cantidad de material en suspensión (Wetzel, 2001), lo que explica que en las estaciones Chico arriba, río Grande y Las Ánimas se presentaran los mayores $\mathrm{K}_{\mathrm{d}(\mathrm{PAR})}$. Incluso las aguas naturales más prístinas no son ópticamente puras, pues invariablemente contienen altas concentraciones de partículas minerales provenientes de la tierra o de los sedimentos del fondo, del fitoplancton, las bacterias, las células muertas y los fragmentos de células, todos los cuales dispersan la luz (Kirk, 2011).
Los $\mathrm{SS}_{\text {tripton }}$ fueron la variable que más aportó al $\mathrm{K}_{\mathrm{d}(\mathrm{PAR}) \text { cal }}$, excepto para la estación Las Ánimas donde el principal aporte lo realizó la $\mathrm{Cl}$. a, con una contribución del 55,0 \%. Según Kirk (1994; 2011), si en el seston, que incluye el fitoplancton y el tripton, el fitoplancton es bajo, entonces el espectro de la fracción particulada puede atribuirse al tripton, lo cual se ajusta a lo hallado en esta investigación. Las propiedades de absorción de la luz por el tripton han recibido poca atención debido a que son difíciles de cuantificar, pues en las concentraciones típicas, este material no absorbe luz sino que la dispersa intensamente, por lo que sus propiedades de absorción no pueden caracterizarse por espectrofotometría normal. Además, el tripton puede limitar la producción de fitoplancton por dos razones: 1) limita la luz disponible para la producción de fitoplancton (Lind, et al., 1992; Phlips, et al., 1995), y 2) dependiendo de su composición puede presentar afinidad por algunos materiales disueltos que, así, dejan de estar disponibles para el fitoplancton (Edzwald, et al., 1976; Mayer, et al., 1987). En la estación Las Ánimas se encontró una baja concentración de tripton, lo que puede beneficiar la producción de fitoplancton, ya que, aunque en poca proporción, impide la penetración lumínica.

Según Tundisi \& Matsumura-Tundisi (2008), en lagos con concentraciones de clorofila entre 10 y $20 \mathrm{mg} / \mathrm{m}^{3}$ la atenuación vertical de la luz es dominada por el tripton, mientras que en lagos y represas con concentraciones de clorofila superiores a $20 \mathrm{mg} / \mathrm{m}^{3}$, como es el caso de la represa estudiada en todas sus estaciones, excepto en la Presa, se presentan coeficientes de atenuación vertical predominantemente influidos por el fitoplancton. No obstante, los datos hallados en esta investigación no concuerdan con esta propuesta, pues como se observa en la tablas 2 y 3 , el fitoplancton como partícula fue el componente que menos aportó al $\mathrm{K}_{\mathrm{d}(\mathrm{PAR})}$ en todas las estaciones. ¿Por qué? Quizás porque la competición de los componentes no vivos del seston (tripton) por la energía radiante limitó la conversión de la energía solar a energía química por parte del fitoplancton, lo cual ocurre cuando las partículas minerales del tripton tienen poco color. Además, como menciona Kirk (1994; 2011), la cantidad de luz cosechada por el fitoplancton depende no sólo de la concentración de los pigmentos fotosintéticos presentes, sino también del tamaño y de la forma de las células o de las colonias de algas que lo contienen. Al respecto, Johnson \& Vahos (2011) encontraron para este embalse un $\mathrm{K}_{\mathrm{s}}$ (coeficiente de sombra dada por las algas) de $0,011 \mathrm{~m}^{2} / \mathrm{mgCl}$. a, muy similar al citado por Kirk (2011) como Kc y usado para calcular $\mathrm{K}_{\mathrm{p} \text {-fito }}(=\mathrm{Cl}$. a.Kc $)$ con un $\mathrm{Kc}$ de $0,014 \mathrm{mgCl} . \mathrm{a} / \mathrm{m}^{2}$. El valor de $\mathrm{K}_{\mathrm{s}}$ hallado por Johnson \& Vahos muestra que por $\mathrm{m}^{2}$ de zona iluminada hay mucha clorofila (media $=392,5 \mathrm{~m}^{2} / \mathrm{mgCl}$. a) que puede impedir la entrada de luz incrementando el valor de $\mathrm{K}_{\mathrm{d}(\mathrm{PAR})}$, lo cual dependerá del tamaño de las algas y de la forma en que estén empaquetadas las unidades de clorofila. Los autores hallaron, además, que en el embalse Ríogrande II 
la clorofila está contenida en células grandes (Ceratium furcoides, Staurastrum paradoxum, Schroederia setigera, Ulnaria ulna), o se reúne en paquetes grandes con células voluminosas en su interior (Microcystis aeruginosa, $M$. wesembergii) que, a pesar de la alta cantidad de clorofila, no consiguen atenuar suficientemente la entrada de luz por los bajos valores de $\mathrm{K}_{\mathrm{s}}$, lo cual confirma la poca importancia del fitoplancton como partícula en la extinción de luz en todas las estaciones del embalse.

Aunque la clorofila a es uno de los principales factores que intervienen en la atenuación de la luz, cada vez se identifican más sistemas en los que los $\mathrm{SS}_{\text {tripton }}$ son los mayores responsables de las variaciones en las características ópticas del agua (Weidemann, et al., 1985; Effler \& Owens, 1985; Effler, et al., 1987; Gerda \& Achterberg, 1992). En un estudio realizado en el lago Okeechobee en Florida, se demostró que el tripton era el constituyente atenuante responsable de las diferencias sustanciales en $\mathrm{K}_{\mathrm{d}}$ de estas cuencas, y de las principales variaciones temporales observadas en dichas características ópticas en la mayoría de las cuencas bajo estudio. Estas partículas inorgánicas llegan directamente desde los afluentes de la cuenca a la columna de agua, particularmente durante las crecientes, o por resuspensión de los sedimentos (Philips, et al., 1995).

Actualmente, varios trabajos cuestionan el valor de la constante $(1,70)$ de Poole \& Atkins (1929), originado a partir del producto Dsd.K ${ }_{\mathrm{d}}$. Vollenweider (1969) estimó este producto como 2.2, Rull, et al. (1984) como variable entre 0,6 y 2,3, y Margalef (1983) entre 1,7 y 2,9. Para Koenings \& Edmundson (1991), el valor de Dsd.K ${ }_{\mathrm{d}}$ osciló entre 1,3 para lagos turbios (como el embalse Ríogrande II) y 3,0 para lagos húmicos, es decir, que su valor es una función del tipo de agua considerada. Para 100 embalses españoles, Margalef, et al., 2003 encontraron que el producto mudaba entre 1,7 y 2,3. Durante el periodo 1995-2001 Armengol, et al. (2003) estimaron en este mismo embalse un valor alternante entre 1,63 (en 1998) y 1,88 (en 1997) para la constante en el intervalo 1997-2001, con un valor medio de 1,78 , muy cercano al citado por la mayoría de los autores como correspondiente a la constante de Poole \& Atkins $(1,7)$. Para el embalse Ríogrande II, se obtuvo una constante de 1,32 (Figura 5), valor similar al hallado en varios embalses catalanes, como el de Susqueda con 1,34, el de Sau con 1,23 y el de Rialb con 1,22, todos en el intervalo de aguas turbias de Koenings \& Edmunson (1991).

Como puede verse en la figura 5, la ecuación obtenida para el cálculo del $\mathrm{K}_{\mathrm{d}(\mathrm{PAR})}$ en el embalse Ríogrande II es de tipo exponencial negativo y bastante cercana a la obtenida por Poole \& Atkins en ecosistemas marinos. En nuestro caso, el $\mathrm{K}_{\mathrm{d}}$ y la Dsd se relacionaron mediante la ecuación $\mathrm{K}_{\mathrm{d}(\mathrm{PAR})}$ $=1,32 \mathrm{Dsd}^{-0,93}$. En esta ecuación consideramos que Dsd explicaba el $62,4 \%$ de los cambios en el $\mathrm{K}_{\mathrm{d}(\mathrm{PAR})}$, y que restaba por explicar un $36,6 \%$, posiblemente debido a otros factores como la turbidez, el color y las sustancias húmicas, los cuales no se evaluaron en esta investigación y de hecho afectan el fenómeno estudiado. No obstante, debe considerarse que este porcentaje de incertidumbre puede deberse también a errores en la medición de Dsd o a la incertidumbre inherente a su estimación. Sin embargo, la pequeña desviación del exponente de la ecuación con respecto a la unidad $(=0,07)$, sugiere que la estimación de dichos factores no mejoraría en mucho la ya lograda.

En muestreos mensuales efectuados en el periodo 20002001 para el embalse de Sau, Armengol, et al. (2003) hallaron valores de $\mathrm{K}_{\mathrm{d}(\mathrm{PAR})}$ entre $0,34 \mathrm{~m}^{-1}$ (en el 2001) y 1,71 $\mathrm{m}^{-1}$ (en el 2000). Para la Bahía de Tampa en Florida, Chen, et al. (2007) proponen un valor de 1,04 $\mathrm{m}^{-1}$. En muestreos realizados durante el verano de 2002 en los 21 embalses españoles, Ordoñez (2003) encontró valores de $K_{d}(P A R)$ que variaron entre $0,3 \mathrm{~m}^{-1}$, en los embalses de Baells, Escales y Siurana, $1,9 \mathrm{~m}^{-1}$ en Terradets, y 3,2 $\mathrm{m}^{-1}$ en el embalse de Foix (valor catalogado como atípico por el autor). En el caso del embalse Ríogrande II, se obtuvo un $\mathrm{K}_{\mathrm{d}(\mathrm{PAR})}$ de $1,32 \mathrm{~m}^{-1}$, relativamente cercano a la mayoría de los valores citados.

\section{Clasificación óptica de Ríogrande II}

Desde el punto de vista óptico, las aguas del embalse Ríogrande II pueden clasificarse en el sistema de Kirk (1980) como pertenecientes al tipo T, es decir aguas en las que la fracción particulada consiste principalmente de material inanimado (tripton), el cual absorbe más luz en todas las longitudes de onda que la fracción soluble; son aguas turbias con grandes cantidades de material suspendido en forma de partículas de lodo y en las que la alta turbidez se debe al tripton y la mayoría de los cuantos son absorbidos por la fracción inorgánica particulada. La clasificación en este tipo óptico se confirma por: 1) la alta turbidez media del sistema (media $=18,8 \pm 13,8 \mathrm{NTU}, \mathrm{n}=$ 35) (Porras-Zapata, et al., 1997), que en el embalse es el factor físico más que la biomasa fitoplanctónica, la cual impide la penetración de la luz; 2) por la mayor contribución del $\mathrm{K}_{\mathrm{d}}$ parcial correspondiente a la fracción de $\mathrm{SS}_{\text {tripton }}$ a la atenuación total de la luz en todas las estaciones $(79,3 \%$ para río Grande, 68,4 \% y 66,3\% para Chico abajo y Chico arriba, respectivamente). Estos porcentajes son similares a los presentados por otros cuerpos de agua del mismo tipo citados por Kirk, $(1994,2011)$, como el lago George en Uganda (fracción soluble: 8,3\%, fracción particulada: 79,3\%), el Georgetown Billabong (fracción soluble: 7,5\%, fracción particulada: 86,6\%) y el embalse de Lake Burley Griffin (fracción soluble: 22,2 \%, fracción particulada: $58,4 \%$ ), y 3 ) por la similitud del valor del producto Dsd. $\mathrm{K}_{\mathrm{dPAR})}=1,32$ en Ríogrande II, con los valores citados por Koenings y Edmundson (1991) para aguas turbias (1,24). Sin embargo, vale la pena considerar que una vez estimada la contribución del $\mathrm{K}_{\mathrm{G}}$ al $\mathrm{K}_{\mathrm{d}}(\mathrm{PAR})$, es posible que el embalse pueda clasificarse como GT. 
En el sistema propuesto por Morel \& Prieur (1977), refinado posteriormente por Gordon \& Morel (1983), las aguas del embalse encajan en el caso 2, en el que el fitoplancton y sus productos derivados pueden o no estar presentes en cantidades significativas y la principal contribución a las propiedades ópticas del cuerpo de agua provienen de los sedimentos resuspendidos desde las orillas del embalse, o de las partículas y el material coloreado disuelto proveniente de la escorrentía de los ríos entrantes o de las descargas industriales. Johnson \& Vahos (2011) hallaron para la razón entre producción y respiración un valor medio de 1,61 , que muestra claramente un desequilibrio entre el material producido por fotosíntesis y el que es eliminado o consumido por la respiración. Esta condición implica que por mucho que el sistema respire, no alcanza a consumir el exceso de entropía que le llega de los alrededores de la cuenca, lo que corrobora el ajuste de las aguas del sistema al caso 2. Vale la pena recordar que la principal práctica económica en la región donde está enclavado el embalse son los cultivos y la explotación lechera y porcina, actividades que demandan insumos que, debido a su manejo inadecuado, contaminan las fuentes de agua del embalse, principalmente con material inorgánico particulado. Además, en el río Chico se practica la minería de aluvión, lo que constituye una fuente importante de sedimentos para esta estación y para el embalse en general.

No obstante, no sobra mencionar que dado que los embalses son sistemas dinámicos, la pertenencia de un cuerpo de agua a uno u otro de los tipos de clasificación óptica establecidos por diferentes autores puede variar.

\section{Conclusiones}

Concluida esta investigación, se acepta parcialmente la previsión efectuada, ya que en la estación río Chico arriba se hallaron los mayores valores del $\mathrm{K}_{\text {tripton }}\left(1,14 \mathrm{~m}^{-1}\right)$ y del $\mathrm{K}_{\mathrm{Cl} \text {. a }}$ $\left(0,49 \mathrm{~m}^{-1}\right)$, pero no valores altos de $\mathrm{K}_{\mathrm{p} \text {-fito }}\left(0,0004 \mathrm{~m}^{-1}\right)$. Como se esperaba, en esta estación se encontró la mayor atenuación vertical de la irradiancia descendente $\left(\mathrm{K}_{\mathrm{d}(\mathrm{PAR})}=1,72 \mathrm{~m}^{-1}\right) \mathrm{y}$ la menor Dsd $(1,06 \mathrm{~m})$ debido a las concentraciones más altas de $\mathrm{Cl}$. a $\left(111,9 \mathrm{mg} / \mathrm{m}^{3}\right), \mathrm{SS}_{\mathrm{T}}\left(16330,5 \mathrm{mg} / \mathrm{m}^{3}\right), \mathrm{SS}_{\text {tripton }}$ $\left(16319,3 \mathrm{mg} / \mathrm{m}^{3}\right), \mathrm{SS}_{\mathrm{p} \text {-fito }}\left(11,2 \mathrm{mg} / \mathrm{m}^{3}\right)$, lo que atenúa la penetración de la luz.

La ecuación para calcular el coeficiente de atenuación vertical de luz en el embalse Ríogrande II fue $\mathrm{K}_{\mathrm{d}(\mathrm{PAR})}=$ $1,32 \mathrm{D}_{\mathrm{sd}}^{-0,93}$. En esta ecuación Dsd explicó el $62,4 \%$ de los cambios en el $\mathrm{K}_{\mathrm{d}}$, restando por explicar un $36,6 \%$ que se debe a otros factores o a la incertidumbre en la medición de Dsd. Dada la poca desviación del exponente de la ecuación con respecto a la unidad $(=0,07)$, consideramos que la estimación de dichos factores no mejoraría en mucho el estimativo alcanzado.

Ópticamente el embalse se ajusta al tipo $\mathrm{T}$ y al caso 2 por ser un cuerpo de agua turbio en el que, a pesar de su condición eutrófica y su alta productividad, no fue el fitoplancton sino el tripton la fracción que más atenuó (por dispersión) la penetración vertical de los quanta fotosintéticos. Finalmente, el $\mathrm{K}_{\mathrm{d}(\mathrm{PAR})}$ calculado para el embalse fue de 1,24 $\mathrm{m}^{-1} \mathrm{y}$ para cada estación fue de $1,01 \mathrm{~m}^{-1}$; de 1,72 y 0,95 para las estaciones Chico arriba y Chico abajo, respectivamente, y para la estación Las Ánimas fue de 1,19, y de $1,16 \mathrm{~m}^{-1}$ para la estación río Grande.

\section{Conflicto de intereses}

Los autores declaran que no tienen conflicto de intereses.

\section{Agradecimientos}

Al Comité de Investigaciones (CODI) de la Universidad de Antioquia por la financiación del proyecto inicial "Caracterización limnológica del embalse Ríogrande II, Antioquia, Colombia" (código 418); a las Empresas Públicas de Medellín (EEPPM) por el apoyo logístico, y al personal de apoyo del embalse por su constante colaboración. Igualmente, a Jaime Ordoñez por sus ideas y su apoyo.

\section{Bibliografía}

American Public Health Association (APHA), American Water Works Association (AWWA), Water Pollution Control Federation (WPCF). (1995). Standard methods for the examination of water and wastewaters. $18^{\text {th }}$ ed. Baltimore: United Book Press.

Armengol, J. L. Caputo, M. Comerma, C. Feijoó, J.C. García, R. Marcé, Navarro, E., Ordóñez, J. (2003). Sau reservoir's light climate: Relationships between Sechhi depth and light extinction coefficient. Limnetica. 22: 195-210.

Clack, P.J \& Williams, F.KL. (1981). Turbidity and suspended solids: What's the difference? Poll. Engin. 13: 43-44.

Baker, K.S. \& Smith, R.C. (1979). Quasi-inherent characteristics of the diffuse attenuation coefficient for irradiance. Soc. Photo-optical Instr. Engin. 208: 60-3.

Bracchini, L., Dattilo, A.M., Hull, V., Loiselle, S.A, Tognazzi, A., Rossi, C. (2009). Modelling Upwelling Irradiance using Secchi disk depth in lake ecosystems. J. Limnol. 68: 83-91.

Chen, Z., Muller-Karger, F.E., Hu, C. (2007). Remote sensing of water clarity in Tampa Bay. En J.T.O Kirk. (2011). Light and photosynthesis in aquatic ecosystems. $3^{\text {rd }}$ ed. (Remote Sensoring Environment 109: 249-59). Cambridge: Cambridge University Press.

Cole, G.A. (1983). Textbook of limnology. The C.V. Saint Louis: Mosby Company.

Davies-Colley, R.J. \& Vant, W.N. (1988). Estimates of optical properties of water from Secchi disk depths. Wat. Res. Bull. 24: 1329-35.

Edzwald J.K., Toensing, D.C., Leung, M.C. (1976). Phosphate adsorption reactions with clay minerals. Environ. Sc. Tech. 10: $485-490$.

Empresas Públicas de Medellín (EEPPM). (1984). Proyecto de aprovechamiento múltiple del Río Grande. Revista Empresas Públicas de Medellín. 6: 172. 
Empresas Públicas de Medellín (EEPPM). (1989). Evolución del desarrollo hidroeléctrico en el departamento de Antioquia. Revista Empresas Públicas de Medellín. 11: 79-81.

Effler, S.W. \& Owens, E.M. (1985). Impact of lake acidification on stratification. Jour. Environ. Engin. Div. ASCE. 111: 822-832.

Effler, S.W, Perkins, M.G., Greer, H. , Johnson, D.L. (1987). Effects of whiting on turbidity and optical properties in Owasco Lake, New York. Wat. Res. Bull. 23: 189-196.

Esteves, F. (2011). Fundamentos de Limnología. Rio de Janeiro: Interciência.

Franco-Velásquez, J.D., Toro-Botero, F.M. , Gómez-Giraldo, A. (2010). Efecto de la posición de la compuerta de captación sobre la estructura térmica de un embalse tropical. XXIV Congreso Latinoamericano de Hidráulica, Uruguay.

Franco-Veláquez, J.D. (2011). Modelación de la estructura térmica de un embalse ramificado mediante el análisis de los procesos físicos gobernantes. Aplicación al embalse multipropósito Ríogrande II (Tesis de Maestría). Universidad Nacional de Colombia. Medellín.

Gerda, M.D. \& Achterberg, E.P. (1992). Light climate in the water column of a shallow eutrophic lake (Lake Veluwe) in the Netherlands. Arch. Hydrobiol. 125: 257-278.

Gordon, H.R. \& Morel, A.Y. (1983). Remote Assessment of Ocean Colour for Interpretation of Satellite Visible Imagery. A Review. Springer, New York. En J.T.O Kirk. (2011). Light and photosynthesis in aquatic ecosystems. $3^{\text {rd }}$ ed. p. 92-95. Cambridge: Cambridge University Press.

Holmes, R.W. (1970). The Secchi Disk in turbid coastal waters. Limnol. Oceanogr. 15: 688-694.

Hutchinson, G.E. (1957). A Treatise on Limnology, Vol. I. New York: Wiley.

Johnson, J. \& Vahos, R. (2011). Clasificación trófica y cinética temporal de las razones $\mathrm{P} / \mathrm{B}, \mathrm{P} / \mathrm{R}, \mathrm{R} / \mathrm{B}$ y $\mathrm{PPN} / \mathrm{PPB}$ en la capa fótica de la presa de un embalse tropical eutrófico (Trabajo de grado). Universidad de Antioquia, Medellín.

Kirk, J.T.O. (1980). Spectral absorption properties of natural waters: contribution of the soluble and particulate fractions to light absorption in some inland waters of southeastern Australia. Aust. J. Mar. Freshwat. Res. 31: 287-96.

Kirk, J.T.O. (1985). Effect of suspensoids (turbidity) on penetration of solar radiation in aquatic ecosystems. Hydrobiologia. 125: $195-208$.

Kirk, J.T.O. (1986). Optical properties of picoplankton suspensions. En T. Platt, \& Li W.K.W. (Eds.). Photosynthetic picoplankton. (pp. 501-520). Can. Bul. Fish. Aq. Sc. 214.

Kirk, J.T.O. (1994). Light and photosynthesis in aquatic ecosystems. $2^{\text {nd }}$ ed. Cambridge: Cambridge University Press.

Kirk, J.T.O. (2011). Light and photosynthesis in aquatic ecosystems. $3^{\text {rd }}$ ed. Cambridge: Cambridge University Press.

Koenings, J. \& Edmundson, J. (1991). Secchi disk and photometer estimates of light regimes in Alaskan lakes:
Effects of yellow color and turbidity. Limnol. Oceanogr. 36: $91-105$.

Lind, O.T., Doyle, R., Vodopich, D.S., Trotter, B.G. Limon, J.G., Davalos, L. (1992). Clay turbidity: Regulation of phytoplankton production in a large, nutrient-rich tropical lake. Limnol. Oceanogr. 37: 549-565.

Loaiza-Restano, A.M., Ramírez, J.J., Echenique, R.O., Vallejo, A., Ortiz, L.Y. (2011). Dinámica espaciotemporal de cuatro especies de Staurastrum (Meyen, 1829; Emend. Ralfs, 1848) en el embalse Ríogrande II (Antioquia), Colombia. Oecol. Austr. 15: 726-746. doi:10.4257/oeco.2011.1503.22.

Londoño, L.F \& Villegas, M.V. (1996). Uso y manejo de plaguicidas en la zona de influencia del embalse Ríogrande II (Trabajo de Grado). Universidad Nacional de Colombia. Medellín.

Margalef, R. (1983). Limnología. Barcelona: Ediciones Omega, S.A.

Margalef, R., Planas, D., Armengol, J. , Vidal, A., Prat, N., Guiset, A., Toja, J., Estrada, A. (1976). Limnología de los embalses españoles. Madrid: Dirección general. Obras Hidráulicas. MOPU.

Mayer, L.M., Nirel, P., Thomas, A.J. (1987). Sequential extraction techniques: Promises and problems. Mar. Chem. 22: $313-341$.

Mazo, D. (2008). Caracterización limnológica del Embalse Ríogrande II (Antioquia, Colombia) (Trabajo de grado). Universidad de Antioquia. Medellín.

Montes-Hugo, M.A., Álvarez-Borrego, S., Giles-Guzmán, A.D. (2003). Horizontal sighting range and Secchi depth as estimators of underwater PAR attenuation in a coastal lagoon. Estuaries. 26: 1302-1309.

Morel, A., \& Prieur, L. (1977). Analysis of variations in ocean colour. Limnol. Oceanogr. 22: 709-22.

Palacio-Betancourt, H. (2014). Dinámica espacio-temporal de las cianobacterias en el embalse Ríogrande II (Tesis de doctorado). Universidad de Antioquia, Medellín.

Phlips, E.J., Aldridge, F.J., Schelske, C.L. Crisman, T.L. (1995). Relationships between light availability, chlorophyll $a$ and tripton in a large shallow subtropical lake. Limnol. Oceanogr. 40: 421.

Poole, H.H \& Atkins, W.R.G. (1929). Photo-electric measurement of submarine illumination troughout the year. J. Mar. Biol. Ass. 16: 297-324.

Porras-Zapata, P., Betancourt-Ángel, A., Molina-Arredondo, A.M., Lopera-Pérez, J.D., Agudelo-García, R.A. (1997). El recurso agua como elemento ordenador del territorio: zona de influencia del embalse Ríogrande II. (Tesis de maestría). Universidad Nacional de Colombia, Medellín.

Preisendorfer, R.W. (1986). Secchi disc science: Visual optics of natural waters. Limnol. Oceanogr. 31: 909-926.

Reynolds, C.S. (1984). The ecology of freshwater phytoplankton. Cambridge: Cambridge University Press. 
Reynolds, C.S. (1979). The limnology of the eutrophic meres of the Shropshire-Cheshire Plain: A review. Field Studies. 5: 93-173.

Roldán, G. \& Ramírez, J.J. (2008). Fundamentos de Limnología Neotropical. 2a ed. Medellín: Academia Colombiana de Ciencias Exactas Físicas y Naturales, Universidad Católica de Oriente, Universidad de Antioquia.

Rull, V., Vegas, T., Navarro, J. (1984). Extinción de la luz en los embalses españoles. Relaciones con la concentración de clorofila y las partículas en suspensión. Oecol. Aq. 7: 2536.

Sartory, D. \& Grobbellar, J. (1984). Extraction of Chloropyll $a$ from freshwater phytoplankton for espectrophotometric analysis. Hydrobiologia. 114: 177-187.

Smith, L.M., Engle, V.D., Summers, J.K. (2005). Assessing water clarity as a component of water quality in Gulf of Mexico estuaries. Environ. Monit. Ass. 115: 291-305.

Tundisi, J. \& Tundisi, T.M. (2008). Limnologia. São Paulo: Oficina de Textos.

Tyler, J.E. (1968). The Secchi disc. Limnol. Oceanogr. 13: 1-6.

Vant, W.N. \& Davies-Colley, R.J. (1984). Factors affecting clarity of New Zealand lakes. New Zeal. J. Mar. Freshwat. Res. 18: $363-77$.
Vant, W.N. \& Davies-Colley, R.J. (1988). Water appearance and recreational use of 10 lakes of the North Island (New Zealand). Verh. Internat. Verein. Limnol. 23: 611-15.

Vergara, A. (2011). Estudio autecológico de Schroederia setigera en un embalse ecuatorial (Trabajo de grado). Universidad de Antioquia. Medellín.

Vollenweider, R. (ed.). (1969). A manual on methods for measuring primary production in aquatic environments. Oxford y Edinburgo: Blackwell.

Weidemann, A.D., Bannister, T.T., Effler, S.W., Johnson, D.L. (1985). Particulate and optical properties during $\mathrm{CaCO}_{3}$ precipitation in Otisco Lake. Limnol. Oceanogr. 30: 10781083.

Weidemann, A.D. \& Bannister, T.T. (1986). Absorption and scattering coefficients in Irondequoit Bay. Limnol. Oceanogr. 31: 567-583.

Wetzel, R.G. (2001). Limnology. W. B. $3^{\text {rd }}$ ed. Philadelphia: Saunders Company.

Zabala, A.M. (2013). Evaluación del estado trófico del embalse Ríogrande II (Antioquia, Colombia) a través del comportamiento espacial y temporal de los nutrientes (Trabajo de grado). Universidad de Antioquia. Medellín. 